\title{
Poison-induced neophobia in rats: Role of stimulus generalization of conditioned taste aversions
}

\author{
MICHAEL DOMJAN \\ University of Texas at Austin, Austin, Texas 78712.
}

\begin{abstract}
Rats repeatedly injected with lithium chloride were subsequently tested drinking novel and familiar solutions of both casein hydrolysate and vinegar. Injections in the absence of edibles result in only a small, and sometimes not reliable, increased avoidance of the novel casein and vinegar solutions. In contrast, if subjects acquired an aversion to saccharin as a result of the lithium injections, this learned aversion generalized to casein hydrolysate, with the generalization greatly enhanced by novelty of the casein flavor. However, the saccharin aversions did not generalize to the novel vinegar solution nearly as much as to the novel casein flavor. These results suggest that previous observations of poison-induced neophobia were probably in part a result of the stimulus generalization of conditioned taste aversions and that in addition to test stimulus novelty some other factor, such as stimulus salience or similarity to the conditioned aversive flavor, is also involved in the generalization of learned taste aversions.
\end{abstract}

Most of the research concerned with the long-term behavioral effects of poisoning in rats has focused on how subjects learn to avoid novel flavored substances experienced before toxicosis. Such poison-avoidance learning has attracted much attention because it occurs even if the novel flavored substance is experienced many hours before poisoning (see review by Revusky \& Garcia, 1970 ) and because it appears to be a good example of adaptation in learning mechanisms (Rozin \& Kalat, 1971).

In addition to motivating the learning of an aversion to novel substances tasted before poisoning, early investigators noted that toxicosis also induces avoidance of other new flavors that were not specifically paired with the aversive agent (Richter, 1953; Rzoska, 1953). Such nonspecific poison-induced neophobia is potentially very important for a complete characterization of the behavioral effects of poisoning, as well as for a better understanding of taste-aversive learning, since the avoidance of substances tasted before toxicosis may be in part poison-induced neophobia. Despite the potential significance of poison-induced neophobia, with few exceptions (e.g., Rozin, 1968), the phenomenon has received little experimental attention after the early observations of Richter and Rzoska.

The present investigation was designed to determine what aspect of a poisoning experience produces flavor neophobia in domesticated rats. to what extent this poison-induced neophobia depends upon a learned taste aversion, and to what extent the neophobia is an instance of stimulus generalization of a conditioned taste aversion.

This research was supported by funds provided by a Biomedical Sciences Support Grant to the University of Texas as well as a grant from the University Research Institute. The uuthor wishes to express appreciation to $C$. Allen and especially K. York for their technical assistance with Experiments I, II, and III $A$. Requests for reprints should be addressed to $M$. Domjan, Department of Psychology, University of Texas at Austin. Austin. 'T'xus 78712

\section{EXPERIMENT I}

The paradigm for observing poison-induced neophobia involves poisoning subjects after exposure to a novel solution " $A$ " and sometime later testing them with a different novel flavor "B." Experiment I sought to determine what aspect of the "Solution A Followed by Poisoning" procedure produces a subsequent reluctance to ingest Solution B. A .2\% sodium saccharin solution served as Solution A and a 5\% casein hydrolysate solution served as B. These two substances were selected because, to the human, they are highly discriminable in terms of both taste and smell. Intraperitoneal injections of lithium chloride were used to produce toxicosis.

\section{Method}

Forty-two 200-250-g naive male Sprague-Dawley rats (Holtzman, Madison, Wisconsin) were individually housed with continual access to Purina Formula Chow and $\mathbf{3 0}$ min daily access to water. Subjects remained in their home cages throughout the experiment except when they were removed for the intraperitoneal injections.

Each subject was assigned to one of four groups after having been on the water deprivation schedule for 3 days. One group $(\mathrm{N}=12)$ received a $20-\mathrm{ml} / \mathrm{kg}$ IP injection of $.12 \mathrm{M}$ lithium chloride immediately after having had access to a $.2 \%$ (weight/volume in tap water) sodium saccharin solution for 10 min (Group Sacc-Li). Another group $(\mathrm{N}=11)$ received the same access to saccharin as Group Sacc-Li but was injected with $20 \mathrm{ml} / \mathrm{kg}$ physiological saline instead of lithium (Group Sacc-Na). The third group $(\mathrm{N}=8)$ received nothing to drink just before being injected with lithium (Group Li), while the fourth group ( $\mathrm{N}=11$ ) was allowed to drink water for $10 \mathrm{~min}$ before being injected with physiological saline (Group $\mathrm{H}_{2} 0-\mathrm{Na}$ ). The treatment given each subject was repeated four times with a 3-4-day interval between successive administrations. On treatment days, the daily $30-\mathrm{min}$ access to water was postponed $1.5 \mathrm{~h}$ after the injections.

A 2-day test sequence was initiated the 3rd day after the fourth treatment. Subjects were tested in a counterbalanced order with tap water and a $5 \%$ (weight/volume) casein hydrolysate solution, both at room temperature. (The casein solution was always prepared just before use in tap water.) On 


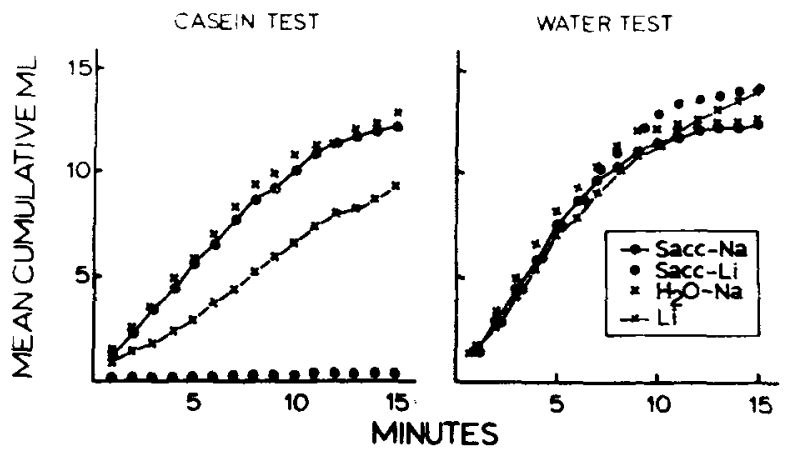

Figure 1. Mean cumulative intake of casein hydroly sate and water in subjects previoushy poisoned after access to saccharin (Group Sacc-Li), poisoned in the absence of edibles (Group Li), not poisoned after access to saccharin (Group Sacc-Na), or neither poisoned nor exposed to saccharin (Group $\mathrm{H}_{2} \mathrm{O}-\mathrm{Na}$ ).

each test day, the test solution (either water or casein) was first presented alone for 15 min in inverted graduated cylinders fitted with stainless steel drinking spouts. The daily 30 -min period of access to water was initiated $30 \mathrm{~min}$ after the end of each day's test. Intakes of the test solutions were recorded at 1-min intervals. All two-group comparisons were made with the Mann-Whitney U test (two-tailed).

\section{Results and Discussion}

Subjects allowed to drink saccharin before each administration of lithium chloride (Group Sacc-Li) quickly learned an aversion to the saccharin flavor. The mean saccharin intake of this group decreased from $13.0 \mathrm{ml}$ on the first injection trial to $0.0 \mathrm{ml}$ on the fourth trial. In contrast, saline-injected control subjects given the identical schedule of access to saccharin (Group Sacc-Na) slightly increased their saccharin intakes from a mean of $12.7 \mathrm{ml}$ on the first trial to $14.3 \mathrm{ml}$ on the fourth trial.

The mean cumulative intake as a function of successive minutes of each test session is presented in Figure 1 for all four groups. No reliable group differences in intake were observed during the test with water. In contrast, during the test with casein, subjects injected with lithium after tasting saccharin (Group Sacc-Li) showed a profound suppression of intake in comparison to each of the other groups (all ps $<.01)$. The two saline-injected control groups $\left(\mathrm{H}_{2} \mathrm{O}-\mathrm{Na}\right.$ and Sacc-Na) drank the most casein and did not differ from each other at any time during the test session. Subjects not given anything to drink just before each lithium injection (Group Li) showed a small suppression of casein intake in comparison to the two nonpoisoned groups. However, assessment of this effect with the Mann-Whitney $U$ test at 5, 10, and 15 min of the test period revealed that Group Li drank reliably less than only Group $\mathrm{H}_{2} \mathrm{O}-\mathrm{Na}$, and this effect was only significant at the 5-min point of the casein test $(\mathrm{p}<.02)$.

In agreement with previous reports, (Carroll, Dinc, Levy, \& Smith, in press; Richter, 1953; Rozin, 1968), the present findings indicate that repeated exposure to the aversive effects of lithium results in reluctance to ingest a novel-flavored solution. Although this neophobia occurred whether or not subjects received access to edibles in conjunction with the lithium injections, exposure to a saccharin solution shortly before each lithium injection greatly increased the subsequent avoidance of the novel casein flavor. The nearly total rejection of the novel casein solution in Group Sacc-Li is rather remarkable since the casein flavor is easily distinguishable from saccharin for humans and was never paired with lithium toxicosis.

\section{EXPERIMENT II}

The greatest reluctance to ingest the novel casein solution in Experiment $I$ was observed in subjects that received access to a saccharin solution shortly before each lithium injection (Group Sacc-Li). Mere exposure to lithium toxicosis probably was only partially responsible for this reaction since subjects that were repeatedly injected with lithium in the absence of edibles (Group Li) subsequently showed a much weaker aversion to the casein flavor. Experiment II was designed to determine whether subjects poisoned after drinking saccharin in Experiment I (Group Sacc-Li) subsequently avoided the novel casein solution also because of the aversion they learned to the saccharin flavor.

\section{Method}

At the end of Experiment 1, Group Sacc-Li was subdivided into an extinction $(\mathrm{N}=6)$ and a control $((\mathrm{N}=6)$ group. On Days 1 and 2 of Experiment II, the extinction group received continuous access to the $.2 \%$ sodium saccharin solution as its only fluid while the control group received continuous access to water. On Day 3, both groups were deprived of all fluids, and on Day 4 they received access to water for $30 \mathrm{~min}$. On Days 5 and 6 , the two groups were given one-bottle tests with the $5 \%$ casein hydrolysate solution and the $.2 \%$ sodium saccharin solution in a counterbalanced order, with the tests conducted as in Experiment I. All two-group comparisons were made with the Mann-Whitney $\mathbf{U}$ test (two-tailed).

\section{Results and Discussion}

The mean cumulative intake as a function of successive minutes of each test session is presented in Figure 2 for each group. The 2-day continuous access to saccharin effectively attenuated the saccharin aversions of the extinction group and resulted in much more saccharin intake during the test sessions in these subjects than was evident in the control group $(p<.01)$. Extinction of the saccharin aversion also attenuated the reluctance of subjects to drink the casein solution. Even though the control group maintained the aversion to casein which was evident in Group Sacc-Li in Experiment I, the extinction group evidenced a greatly elevated casein intake in comparison $(p<.01)$.

The present results indicate that the suppression of casein intake observed in subjects injected with lithium 
aller drinking saccharin is very much dependent upon the extent (1) which these subjects avoid the saccharin falvor. The learned aversion to saccharin appears to mediate avoidance of the casein solution. This mediation may reflect the possibility that the aversion to casein is a poison-induced neophobia reaction which is enhanced by taste aversions conditioned by the poisoning experience. (Such a hypothesis would also explain why Group Li in Experiment I did not show as strong an aversion to the novel casein solution as Group Sacc-Li.) Alternatively, perhaps subjects that have a learned aversion to saccharin also avoid drinking casein because the taste of casein is similar to that of saccharin and there is a stimulus generalization of the aversion. Although the casein and saccharin solutions are highly discriminable to humans, to the rat the two substances may have certain elements in common.

\section{EXPERIMENT III}

Experiment III was designed to determine whether subjects injected with lithium after drinking saccharin subsequently avoid drinking a casein solution because of its novelty or because of its similarity to saccharin. Independent groups injected with lithium after drinking saccharin were subsequently tested with each of two flavors, a $3 \%$ concentration of vinegar and a $5 \%$ concentration of casein hydrolysate, in a counterbalanced order. For half the subjects, the vinegar flavor was novel, and the casein was familiar; for the other subjects, casein was novel, and the vinegar flavor was familiar (see Table 1). If subjects with a learned aversion to saccharin subsequently avoid another test solution because of its novelty, the group familiarized with casein would be expected to avoid drinking the vinegar solution, while the group familiarized with vinegar would be expected to avoid drinking the casein flavor. In contrast, if the avoidance of test fluids depends upon their similarity to saccharin, comparable avoidance reactions to both casein and vinegar would not be expected since it is highly unlikely that both of these solutions are equally similar to saccharin.

In Experiment IIIA, casein- and vinegar-familiarized

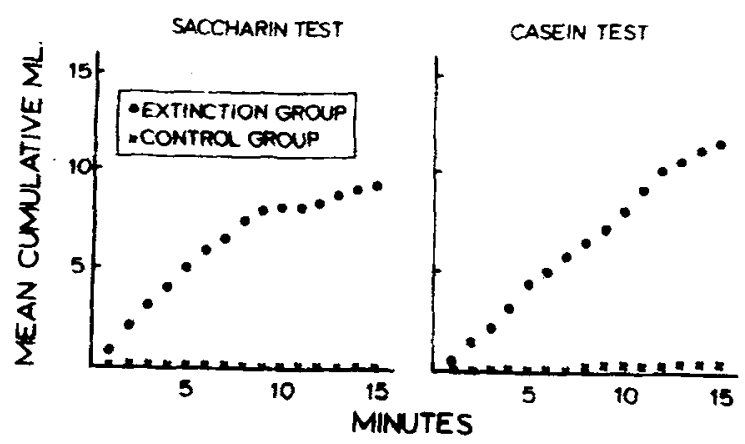

Figure 2. Mean cumulative intake of saccharin and casein in extinction and control groups of Experiment II.
Table 1

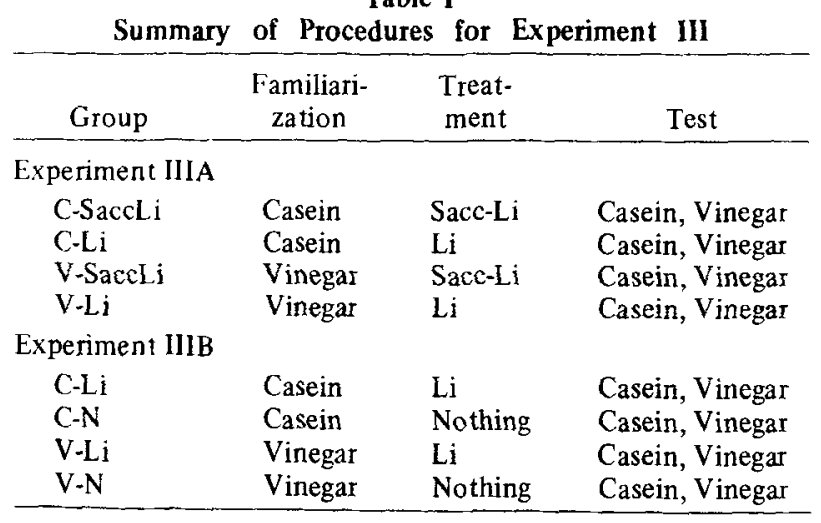

subjects injected with lithium after drinking saccharin were compared to casein- and vinegar-preexposed subjects that received only the lithium injections. In Experiment IIIB, subjects that received only the lithium injections were compared to a noninjected control group to determine whether these injections alone produced flavor neophobia under conditions of the present experiment. The full design of the experiment is summarized in Table 1.

\section{Method}

Experiment IIIA. Thirty-seven male albino offspring of Sprague-Dawley rats (Holtzman, Madison, Wisconsin) were born and reared on continual access to Purina Formula Chow and water in the University of Texas laboratories. The subjects were restricted to a daily $30-\mathrm{min}$ period of access to water when their mean weight reached $252 \mathrm{~g}$.

Familiarization training was initiated after subjects had been on the wate deprivation schedule for 3 days. For 19 subjects, a $3 \%$ (volume/volume) solution of apple cider vinegar was substituted for water during the daily $30-\mathrm{min}$ fluid access periods. For the remaining 18 subjects, a $5 \%$ (weight/volume) casein hydrolysate solution was substituted for water. This familiarization training with the vinegar and casein solutions was continued for 8 consecutive days.

Subjects were then assigned to one of two injection treatments comparable to the Sacc- $\mathrm{Li}$ and $\mathrm{Li}$ conditions tested in Experiment $I$. Ten of the vinegar-familiarized subjects and nine of the casein-familiarized subjects were given $20 \mathrm{ml} / \mathrm{kg}$ IP injections of $.12 \mathrm{M}$ lithium chloride immediately after having had access to a $.2 \%$ (weight/volume) sodium saccharin solution for $10 \mathrm{~min}$ (Groups V-SaccLi and C-SaccLi, respectively; the first letter of the group designations indicates the solution to which subjects were preexposed.) The remaining vinegar- and casein-familiarized subjects were treated identically except they did not receive anything to drink just before the lithium injections (Groups V-Li and C-Li). The injections were administered 3 times at $48 \mathrm{~h}$ intervals. As in Experiment $\mathrm{I}$, on days when they were administered lithium, subjects received their daily $30-\mathrm{min}$ access to water $1.5 \mathrm{~h}$ after the injections.

A 2 -day test sequence was initiated $48 \mathrm{~h}$ after the third lithium injection. Subjects received 15 -min one-bottle tests with the $3 \%$ vinegar and $5 \%$ casein solutions in a counterbalanced order, with the tests conducted as in Experiment I. Immediately after each day's test, tap water was available for $30 \mathrm{~min}$.

Experiment IIIB. Thirty-six male 200-250-g Sprague-Dawley rats (Holtzman, Madison, Wisconsin) were used. The method was identical to that of Experiment IIIA in all unspecified details. Eighteen subjects were familiarized with the $5 \%$ casein hydrolysate solution while the remaining 18 were familiarized 
Table 2

Mean Milliliters of Saccharin Ingested During Successive Conditioning Trials in Experiment IIIA

\begin{tabular}{lccc}
\hline Group & Trial 1 & Trial 2 & Trial 3 \\
\hline C-SaccLi & 12.1 & 3.0 & .1 \\
V-SaccLi & 11.9 & .8 & .0 \\
\hline
\end{tabular}

with the $3 \%$ vinegar solution. Nine subjects in each of these preexposure conditions were then injected with lithium chloride on three ofcasions as Groups V-Li and C-Li of Experiment IIIA. The remaining nine subjects in each familiarization condition were treated identically except no injections were given (Groups $\mathrm{V}-\mathrm{N}$ and $\mathrm{C}-\mathrm{N}$; "N" for "no-injection"). The one-bottle tests with the casein and vinegar solutions were initiated 1 day after the third lithium injection and were conducted as in Experiment IIIA. All two-group comparisons were made with the Mann-Whitney U test (two-tailed).

\section{Results}

Experiment IIIA. The amount of saccharin ingested during successive conditioning trials by subjects allowed access to this solution just before each lithium injection is shown in Table 2. Before the first injection, vinegarand casein-familiarized groups (V-SaccLi and C-SaccLi) drank comparable amounts. As expected, both groups decreased their saccharin intakes from the first to the second conditioning trial, indicating that the first lithium injection induced an aversion to the saccharin flavor. The reliably lower saccharin intakes in Group V-SaccLi as compared to Group C-SaccLi on the second trial $(p<.02)$ suggests that the vinegar-familiarized subjects learned a slightly stronger saccharin aversion as a consequence of the first lithium injection than the casein preexposed subjects. However, this small difference was probably overcome by the second and third trials since during the third trial both vinegar- and casein-familiarized subjects almost totally avoided the saccharin solution.

The mean cumulative intake as a function of successive minutes of each test session is presented in Figure 3 for each group. During the test with vinegar, the only variable which influenced the amount ingested was the flavor with which subjects were familiar. Those preexposed to the vinegar solution (Groups V-SaccLi and V-Li) drank reliably more than those familiarized with casein (Groups C-SaccLi and C-Li) for whom the vinegar solution was novel $(p<.01)$. Access to saccharin just before the lithium injections had no effect on vinegar intake. Group V-SaccLi drank as much vinegar as Group V-Li and Group C-SaccLi drank as much as Group C-Li.

During the test with casein, novelty of the test solution again suppressed intakes. Subjects injected with lithium after drinking saccharin, for whom the casein solution was novel (Group V-SaccLi), drank much less than similarly treated subjects for whom the casein solution was familiar (Group C-SaccLi, p<.01). Correspondingly, Group V-Li, for whom the casein solution was novel, drank reliably less than Group C-Li which was familiar with casein $(\mathrm{p}<.02)$.

In contrast to the results obtained during the vinegar tests, access to saccharin just before each lithium injection also affected subsequent intakes of the casein solution. Subjects repeatedly poisoned after drinking saccharin drank less casein that subjects which had been poisoned in the absence of edibles. This effect was highly reliable with subjects for whom the casein solution was novel (Groups V-SaccLi vs. V.Li, p <.01) but did not reach conventional levels of significance when the casein solution was familiar (Groups C-SaccLi vs. C-Li).

Experiment IIIB. The results of each test session are presented in Figure 4. During both the test with vinegar and the test with casein, the only variable which affected intakes was the noveity of the test solution. Subjects familiarized with vinegar (Groups V-Li and V-N) drank reliably more of this substance than subjects familiarized with casein $(p<.01)$, whereas subjects familiarized with casein (Groups $\mathrm{C}-\mathrm{Li}$ and $\mathrm{C}-\mathrm{N}$ ) drank significantly more casein than those preexposed to vinegar (Groups V-Li and V.N, $p<.01$ ). Lithium-injected subjects (Groups $\mathrm{C}$ - Li and $\mathrm{V}-\mathrm{Li}$ ) did not drink less than the corresponding noninjected control subjects (Groups C-N and V-N) in any of the test conditions.

\section{Discussion}

In each of the lithium-injection conditions (SaccLi and $\mathrm{Li}$ in Experiment IIIA and $\mathrm{Li}$ in Experiment IIIB), as well as in the no-injection control condition ( $\mathrm{N}$ in Experiment IIIB), intakes of the casein and vinegar test solutions were less when the solutions were novel than when they were familiar. These results indicate that the casein and vinegar flavors elicited neophobia. However, in the present experiment, this neophobia was not

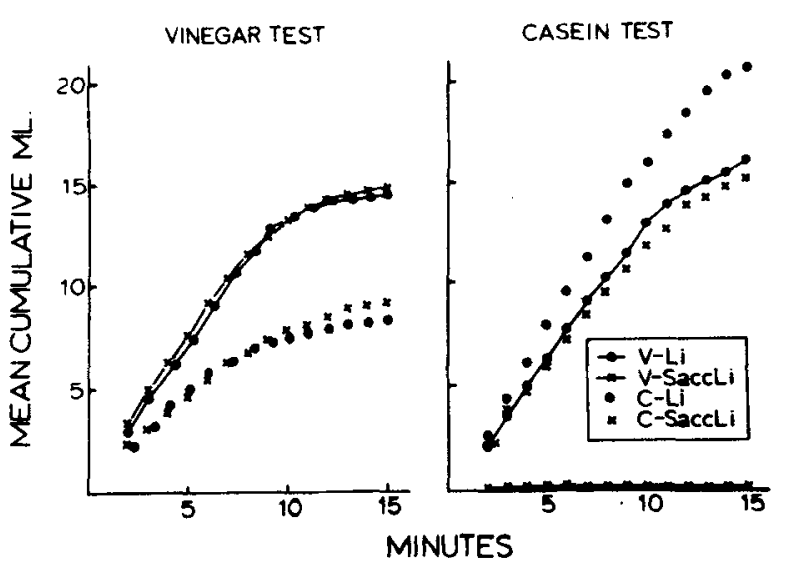

Figure 3. Mean cumulative intake of vinegar and casein in vinegar-familiarized subjects (Groups V-Li and V-SaccLi) and casein-familiarized subjects (Groups $\mathrm{C}-\mathrm{Li}$ and $\mathrm{C}$-SaccLi) previously poisoned either in the absence of edibles (Groups V-Li and $\mathrm{C} \cdot \mathrm{Li}$ ) or after access to saccharin (Groups V-Saccli and C-SaccLi) in Experiment IIIA. 
reliably enhanced by prior experience with lithium injections in the absence of edibles (Experiment IIIB, Figure 4). This outcome suggests that the small enhanced neophobia observed with Group Li in Experiment I does not represent a very robust phenomenon.

As in Experiment I, subjects that acquired an aversion to saccharin because of the repeated lithium injections subsequently showed much greater reluctance to drink the novel casein solution than subjects previously injected with lithium in the absence of edibles (Group V-SaccLi as compared to Group V-Li). In fact, this generalized learned aversion from saccharin to casein resulted in nearly total rejection of the novel casein flavor in Group V.SaccLi. Furthermore, the aversion appeared to be in part a response to the novelty of the casein solution since subjects for whom casein was familiar (Group C-SaccLi) drank much more casein than those unfamiliar with the solution (Group V-SaccLi), and the intakes of casein-familiarized subjects (Group C-SaccLi) were not reliably different from controls that did not previously experience saccharin aversion conditioning (Group C-Li). These findings suggest that novelty of a test solution facilitates the generalization of a conditioned taste aversion.

In contrast to the nearly complete stimulus generalization of the conditioned saccharin aversion to the novel casein solution, much less generalization occurred between the aversive saccharin flavor and the novel vinegar solution. This generalization was not sufficient to produce less intake of the novel vinegar solution following saccharin aversion conditioning (Group C.SaccLi) than following lithium-only treatments (Group C-Li). Furthermore, subjects given saccharin aversion conditioning subsequently drank much more of the novel vinegar solution (Group C-SaccLi, mean $=9.1 \mathrm{ml}$ ) than of the novel casein solution (Group V-SaccLi, mean $=.1 \mathrm{ml}, \mathrm{p}<.01$ ). These findigns suggest that novelty of a test solution is not the only stimulus variable involved in the generalization of taste aversions.

It may be argued that the acquired aversion to saccharin generalized more readily to the novel casein than to the novel vinegar solution because the novel vinegar solution was not as unfamiliar to the subjects. Unfamiliarity may be measured by how much subjects drink of a solution on first encounter or by the extent to which novelty of a solution suppresses intake in comparison to how much is ingested when the solution is familiar. According to the first measure, the vinegar solution was more novel than the casein solution because on first encounter noninjected control subjects drank only a mean of $9.8 \mathrm{ml}$ vinegar whereas they drank a mean of $15.7 \mathrm{ml}$ of the casein solution (see Figure 4). According to the second measure, the two solutions were comparably unfamiliar. The mean casein intake of nonpoisoned subjects unfamiliar with casein (Group V-N) was $67 \%$ of the mean casein intake of

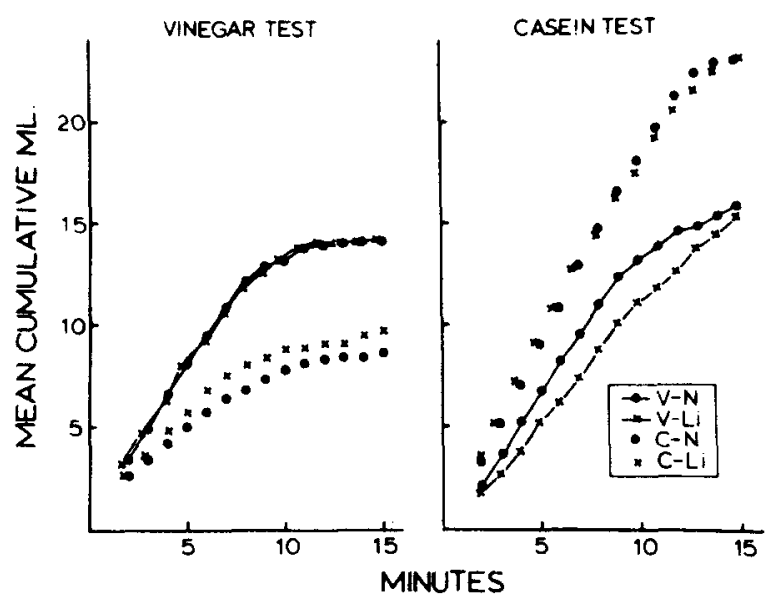

Figure 4. Mean cumulative intake of vinegar and casein in vinegar-familiarized subjects (Group V-Li and V-N) and casein-familiarized subjects (Groups $\mathrm{C}-\mathrm{Li}$ and $\mathrm{C}-\mathrm{N}$ ) previously either not poisoned (Groups V-N and $\mathrm{C}-\mathrm{N}$ ) or poisoned in the absence of edibles (Groups V-Li and C-Li) in Experiment IIIB.

casein-familiarized subjects (Group $\mathrm{C}-\mathrm{N}$ ), while mean intake of the novel vinegar flavor (Group C-N) was $67 \%$ of the mean intake of vinegar when vinegar was familiar (Group V.N). Given these results, it seems unlikely that the learned saccharin aversions generalized much more to the novel casein than vinegar solutions because the novel casein solution was much more unfamiliar.

\section{EXPERIMENT IV}

Conditioned aversions to saccharin in Experiment III generalized much more readily to a novel than to a familiar casein solution. Although this outcome was probably due to the differences in novelty of the casein flavor, differences in saccharin aversion learning also may have been involved since familiarization with casein slightly interfered with conditioning an aversion to the saccharin solution. To minimize the possible contribution of such interference with saccharin aversion learning, in Experiment IV subjects familiarized with casein were conditioned to have a stronger saccharin aversion before the generalization tests than subjects for whom the casein solution was novel. In addition, the extent to which conditioned aversions to saccharin generalize to novel casein and vinegar flavors was directly compared using a single group of subjects not previously exposed to either test solution.

\section{Method}

Thirty-two naive male Sprague-Dawley rats $(200-250 \mathrm{~g})$ were used in procedures identical to those of Experiment III in all unspecified details. Sixteen subjects received access to the 5\% casein hydrolysate solution for $30 \mathrm{~min}$ daily over an 8-day period, while the remaining 16 subjects were maintained on tap water $30 \mathrm{~min} / \mathrm{day}$. At the end of this period, eight of the case in-familiarized subjects received two saccharin-lithium aversion conditioning trials (Group C-2SLi) while the remaining eight casein-familiarized subjects received no exposure to either saccharin or lithium toxicosis (Group $\mathrm{C}-\mathrm{N}$ ). Half of the subjects 


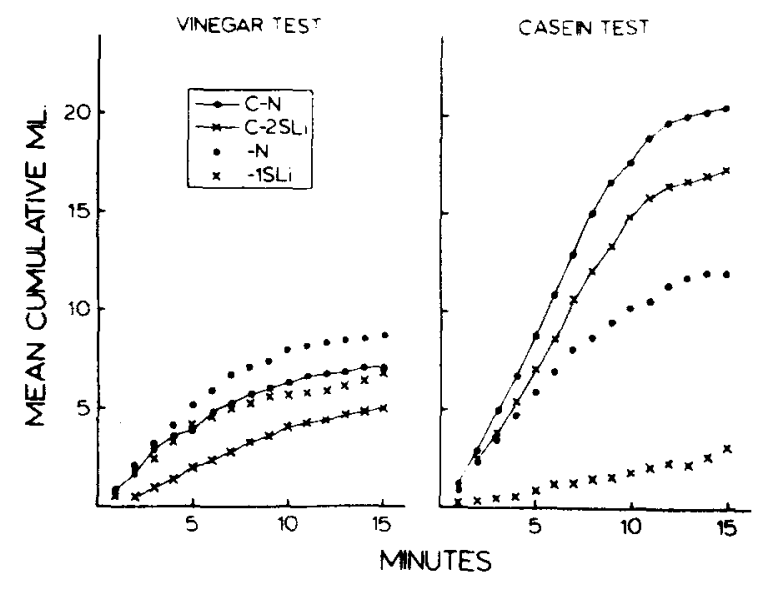

Figure 5. Mean cumulative intake of vinegar and casein in casein-familiarized subjects (Groups $\mathrm{C}-2 \mathrm{SLi}$ and $\mathrm{C}-\mathrm{N}$ ) and nonpreexposed subjects (Groups $-1 \mathrm{SLi}$ and $-\mathrm{N}$ ) previously given either $\mathrm{O}$ (Groups $\mathrm{C}-\mathrm{N}$ and $-\mathrm{N}$ ), 1 (Group 1SLi), or 2 (Group C-2SLi) saccharin aversion conditioning trials in Experiment IV.

not familiarized with casein also received aversion conditioning to the taste of saccharin, but they only experienced one conditioning trial (Group-1SLi). The remaining eight nonfamiliarized subjects received no exposure to either saccharin or toxicosis (Group-N). Two days after this differential treatment, all subjects were tested in a counterbalanced order with $5 \%$ casein and $3 \%$ cider vinegar as in Experiment III. In addition, subjects received a $15-\mathrm{min}$ one-bottle test with the saccharin solution the day after the case in and vinegar tests. As in previous experiments, two-group comparisons were made with the Mann-Whitney U test (two-tailed).

\section{Results}

As expected, both of the groups injected with lithium chloride after access to saccharin acquired aversions to the saccharin flavor. During the postconditioning test with saccharin, Group -1SLi drank less than its corresponding control group, Group -N $(p<.01)$, and Group C-2SLi drank less than its control group, Group C-N $(p<.01)$. Furthermore, this learned aversion was greater in subjects given two conditioning trials (Group C-2SLi) than in subjects poisoned only once after exposure to saccharin (Group-1SLi), despite the extensive prior exposure of Group C-2SLi to casein hydrolysate. Group C-2SLi drank reliably less saccharin during the postconditioning tests (mean $=0.0 \mathrm{ml}$ ) than Group - $1 \mathrm{SLi}($ mean $=2.5 \mathrm{ml}, \mathrm{p}<.01)$.

The amount of vinegar and casein ingested by each group during the test sessions is presented in Figure 5. Groups conditioned to have an aversion to saccharin did not drink reliably less vinegar than their corresponding noninjected controls. That is, neither the difference between Groups $\mathrm{C}-2 \mathrm{SLi}$ and $\mathrm{C}-\mathrm{N}$ nor the difference between Groups $-1 \mathrm{SLi}$ and $-\mathrm{N}$ in vinegar intake reached conventional levels of statistical significance. In contrast, saccharin aversion conditioning produced reliable suppressions in casein drinking. Group C-2SLi drank less casein than Group C-N $(p<.05)$ and Group -1SLi drank less than Group-N $(p<.01)$. However, as in Experiment III, the generalized aversion to the casein solution was much more pronounced in subjects for whom the casein flavor was novel than in subjects given prior familiarization with casein. Group -1SLi drank a mean of $8.7 \mathrm{ml}$ less than Group $\cdot \mathrm{N}$ during the test with case in whereas Group C-2SLi drank only a mean of $3.2 \mathrm{ml}$ less than Group C-N. Furthermore, the difference in casein intake between Groups $-1 \mathrm{SLi}$ and $-\mathrm{N}$ was also reliable at $10 \mathrm{~min}$ of the casein test $(p<.01)$ while the difference between Groups C-2SLi and $\mathrm{C}-\mathrm{N}$ was not reliable at this point.

In addition to the between-subject comparisons discussed above, the present experiment also made possible a within-subject comparison of the extent to which conditioned aversions to saccharin generalized to the novel vinegar and casein flavors. Group - $1 \mathrm{SLi}$, for which both the casein and vinegar test solutions were novel, drank reliably more of the vinegar than the casein solution after acquiring an aversion to saccharin (sign test $x=0, p<.01)$. In contrast, in the absence of aversion conditioning, subjects (Group -N) tended to drink more of the novel casein than vinegar solution, although in this experiment this difference was not reliable $(x=3, p>.05)$.

\section{Discussion}

The results of the present experiment show that an acquired aversion to the flavor of saccharin generalizes more readily to a novel than a familiar casein solution even if casein-preexposed subjects have stronger saccharin aversions than subjects for whom the casein flavor is novel. This finding confirms that the weaker generalized aversion to a familiar than a novel casein flavor observed in Experiment III was due to differences in novelty of the casein solution rather than the possibly weaker saccharin aversions learned by casein-preexposed subjects.

The present results also indicate that conditioned aversions to saccharin generalize much more to a novel casein than a novel vinegar flavor. This outcome confirms a similar finding in Experiment III and shows that such results can be obtained using a within-subject design without the confounding which existed in Experiment III between the novelty of one test solution and familiarity of the other. These results therefore lend further support to the conclusion that test stimulus novelty is not the only stimulus variable which influences the generalization of conditioned taste aversions.

\section{GENERAL DISCUSSION}

The present experiments indicate that lithium injections that do not condition a taste aversion produce only a smail, and sometimes not reliable, increased avoidance of novel-tasting substances. In contrast, if the 
injections are paired with a distinctive taste and condition a taste aversion, this learned aversion generalizes to other flavors, and the generalization is greatly enhanced by novelty of the test solutions. However, novelty did not appear to be the only stimulus variable involved in taste aversion generalization since learned aversions did not generalize equally to all novel flavors.

In addition to test stimulus novelty, stimulus generalization of conditioned taste aversions may also depend upon the extent of similarity between the test solutions and the conditioned aversive flavor. 1 In the present study, conditioned aversions to saccharin may have generalized much more to the novel casein than the novel vinegar flavor because, of these two solutions, casein is more similar to saccharin for rats. Such an assumption also explains why preexposure to the casein solution interfered slightly with the acquisition of a taste aversion to saccharin in Group C-SaccLi of Experiment IIIA (see Table 2). A number of studies have shown that preexposure to a taste solution interferes with subsequent taste-aversion conditioning (e.g., Domjan, 1972). If two solutions are similar, this interference effect would be expected to generalize from one to the other.

The notion that generalization of a learned taste aversion depends upon both a certain degree of similarity between the test stimulus and the conditioned aversive flavor, as well as novelty of the test stimulus, is consistent with both the early poison-induced neophobia reports of Richter (1953) and Rzoska (1953) as well as the recent findings of Carroll et al. (in press) but appears inconsistent with Rozin's (1968) observation that generalization requires only test stimulus novelty. However, as has been pointed out earlier (Brackbill, Rosenbush, \& Brookshire, 1971), Rozin's evidence was obtained when subjects may have been still experiencing the effects of poisoning and therefore cannot be regarded as a long-term behavioral reaction to toxicosis. (Neophobia for flavored solutions in rats suffering from poisoning has also been observed by Barker, Suarez, \& Gray, 1974.) Furthermore, test stimulus novelty may be seen as the only factor determining the generalization of learned aversions if the test stimuli used are all about equally similar to the conditioned aversive flavor.

An alternative to explaining the present results in terms of two factors, novelty and similarity, determining the stimulus generalization of learned taste aversions may be to consider a single process, stimulus salience (Kalat \& Rozin, 1970) or associability with toxicosis, as the only variable in taste aversion generalization. It has often been observed that novelty increases the salience or associability of a stimulus with toxicosis (e.g., Domjan, 1972; Kalat, 1974). In the present study, perhaps the acquired saccharin aversions generalized more readily to a novel than to a familiar casein solution because the rovel casein flavei was more salient. Similarly, the learned aversions may have generalized to a greater extent to casein than to vinegar because the casein flavor was more salient than the taste of vinegar. In addition to being consistent with the present results, this single-process view of taste-aversion generalization is also consistent with the previous observations of poison-induced neophobia.

\section{REFERENCES}

Barker, L. M., Suarez, E. M., \& Gray, D. Backward conditioning of taste aversion in rats using cyclophosphamide as the US Physiological Psychology, 1974, 2, 117-119.

Brackbill. R. M., Rosentush, S. M., \& Brookshire, K. H. Acquisition and retention of conditioned taste aversions as a function of the taste quality of the CS. Learning and Motivation, 1971, 2, 341-350.

Carroll, M. E., Dinc, H. I., Levy, C. J., \& Smith, J. C. Demonstrations of neophobia and enhanced neophobia in the albino rat. Journal of Comparative and $P$ hysiological Psychology, in press, 1975

Domjan, M. CS preexposure in taste aversion learning: Ef fects of deprivation and preexposure duration. Learning and Motivation, $1972,3,389 \cdot 402$.

Kalat, J. W. Taste salience depends on novelty, not concentration, in taste-aversion learning in the rat. Jourmal of Comparative and Physiological Psychology, 1974, 86, 47-50.

Kalat, J. W., \& Rozin, P. "Salience": A factor which can override temporal contiguity in taste aversion learning. Joumal of Comparative and Physiological Psychology, 1970, 71, 192-197.

Revusky, S. H., \& Garcia, J. Learned associations over long delays. In G. H. Bower and J. T. Spence (Eds.), The psychology of learning and motivation: Advances in research and theory (Vol. 4). New York: Academic Press, 1970.

Richter, C. P. Experimentally produced bebavior reactions to food poisoning in wild and domesticated rats. Annals of the New York Academy of Sciences, 1953, 56, 225-239.

Rozin, P. Specific aversions and neophobia resulting from vitamin deficiency or poisoning in half-wild and domestic rats. Journal of Comparative and Physiological Psychology, 1968 , 66, 82-88.

Rozin, P., \& Kalat, J. W. Specific hungers and poison avoidance as adaptive specializations of learning. Psychological Review, $1971,78,459-486$

Rzoska, J. Baity shyness, a study in rat behavior. British .Journal of Animal Behavior, 1953, 1, 128-135.

\section{NOTE}

1. It is important to note that similarity between two flavors does not imply that they are indiscriminable. Two flavors may be easily distinguishable and still have certain common elements.

(Received for publication August 20, 1974; revision accepted January 15,1975 .) 